\title{
Raining Down on Rising Sea Levels
}

\author{
Team Number: 8597
}

February 2017 


\section{Executive Summary}

The National Park Service (NPS) is committed to preserving the beauty of America in order to provide everyone amazing interactions with nature. For over 100 years, the NPS has maintained these wonders of America; however, as it begins its second century of operation, one of the NPS's greatest concerns is the issue of climate change. Climate change greatly influences actions the NPS takes to protect parks and events such as flooding or other disasters can affect how many people visit the park.

In particular, rising sea levels are one of the imminent problems that the United States is faced with because of its impact on flooding, and it is necessary for the NPS to identify which National Parks are at risk. We were initially tasked with developing a model to classify 5 particular parks as having either high, medium, or low risk of sea level change. For each location, we created a probabilistic model of the sea level height in the next $t$ years. We determined whether a site had high, medium, or low risk levels based on the damage that we would expect to occur based on the change in sea level. We calculated the probability of risk associated with each region in 10, 20, 50, and even 100 years from now. Our findings show that Cape Hatteras and the Padre Island possess the greatest risk of all 5 national parks.

After classifying these parks as high, medium, or low risk based on sea level change alone, we sought to determine a set of additional criteria to build a model that would assign each site a "vulnerability score." The vulnerability score is based off of the likelihood and severity of climate related events occurring. We selected our criteria to be the Heat Index, which consisted of temperature and humidity, hurricane intensity and frequency, and the Air Quality Index. These criteria were then used to construct a model that generated the Vulnerability score by first assigning a subscore for each of the individual criteria and then taking a weighted average of these subscores. We found that Padre Island National Seashore and Acadia National Park are in critical condition, with Padre Island being in a worse condition than Acadia National Park. Furthermore, Olympic National Park and Cape Hatteras National Seashore are still safe but almost in a critical condition, and Kenai Fjords National Park is the safest of all five.

Finally, we created a model to determine how to allocate limited funds to the parks based off of factors including the adjusted vulnerability score we calculated in part two, as well as the number of visitors for each park. We accomplished this by first determining the expected number of visitors for the future based on data from previous years and the vulnerability score. We then used the results of our model to convert our predictions of the number of visitors and the vulnerability scores into indices that would calculate the overall Financial Utility index. We used the financial utility indices for each site to decide the optimal distribution of funds between the 5 parks. Based on our results, we found that the percentage of funds should be allocated as follows from most to least: Acadia National Park (30.48\%), Olympic National Park (28.27\%), Cape Hatteras (21.49\%), Padre Island (10.94\%), Kenai Fjords (8.82\%). 


\section{Contents}

1 Executive Summary

2 Part 1: Water We Going to Do?

2.1 Restatement of the Problem

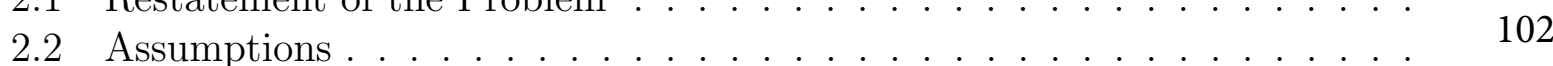

2.3 Developing the Model . . . . . . . . . . . . . . . . . . . . . . . . . . . . . . . . . . . . . . .

2.4 Validity of Model and Generalities . . . . . . . . . . . . . 105

3 Part 2: Modeling the Effects of Heat Index, Air Quality, and Hurricane 106 Frequency and Intensity on Vulnerability Scores 106

3.1 Restatement of the Problem . . . . . . . . . . . . . . . . . . 106

3.3 Developing the Model . . . . . . . . . . . . . . . . . . . . . . . 107

3.4 Putting it all together . . . . . . . . . . . . . . . . . . . 108

3.5 Verifying the Model . . . . . . . . . . . . . . . . . . . . . 109

3.6 Future Projections . . . . . . . . . . . . . . . . . 110

4 Part 3: Profitability and Vulnerability 112

4.1 Restatement of the Problem . . . . . . . . . . . . . . . . . . . . 112

4.2 Analysis of the Problem . . . . . . . . . . . . . . . . . 112

4.3 Assumptions . . . . . . . . . . . . . . . . . . . . . . . . . . 112

4.4 Developing the Model . . . . . . . . . . . . . . . . . . . . . . . 113

4.5 Justification and Discussion . . . . . . . . . . . . . . 115

5 Conclusion $\quad 116$

5.1 Strengths . . . . . . . . . . . . . . . . . . . . . . . . . . . . . . . . . . .

5.2 Weaknesses ... . . . . . . . . . . . . . . . 116

6 References 117 


\section{Part 1: Water We Going to Do?}

\subsection{Restatement of the Problem}

The problem asks us to do the following:

- Build a mathematical model to determine the change in sea level for each of the following five parks:

Acadia National Park, Maine

Cape Hatteras National Seashore, North Carolina

Kenai Fjords National Park, Alaska

Olympic National Park, Washington

Padre Island National Seashore, Texas

- Rate each of these National Parks as one of high, medium, or low risk of sea level change for the next 10, 20, and 50 years.

- Determine whether our model could be used to make a prediction for the next 100 years.

\subsection{Assumptions}

- The sea level rate of change found over the past 100 years will continue to be constant over the next 100 years as well.

Justification: Using data from the National Oceanic and Atmospheric Administration [1], we can consider the consistency in sea level rate change due to no statistically significant difference between the calculated trends of change in sea level. Because the $95 \%$ confidence intervals depicting sea level overlap for mean sea level trends over the past 100 years, for all 5 sites, we can extrapolate a similar trend for at least short run models under a few centuries.

- Increasing sea level is directly correlated with increasing risk of flood.

Justification: While it is true that as sea levels increase the local government is more inclined to build reinforcements to minimize damage it is obvious that risk of flood is highest when the sea level is highest.

- All sites start at a baseline of having no predisposed greater risk than another

Justification: Evidently, some sites are more likely to be favored more than others from the start for being safer or riskier than others. However, randomness in variables contributing towards Risk cannot be totaled and in creating a general model to apply to any site, we limit it to ubiqutuous factors.

- Temperature will not affect our model on sea level change over the duration over where are model operates.

Justification: While the NOAA accounts for 2 factors that play a roll in increasing sea levels: thermal expansion and increased melting of land based ice 
[19], temperature can still be discounted. Plotting average yearly temperature over roughly 20 years across each site, linear, polynomial, and lograthmic lines of best fit all fail to exceed an $R^{2}$ value of 0.1 . Because not even $0.1 \%$ of difference in the yearly temperature across 2 decades can be accounted for by any time, we can discount the effect of temperature as it's simply unpredictable [8]. This inability to predict any temperature across such a short span of few years is only resolved by comparing a few of centuries at a time. Thus, despite thermal expansion and increased melting of land based ice being directly proportional to change in temperature, temperature change only needs to be factored into models running over a few centuries which ours does not.

\subsection{Developing the Model}

We begin our model by analyzing the average change in mean sea level of all the five sites and the standard deviation of each site as well. In doing so, we hope to be able to predict the likelihood of the sea level of each site rising above certain benchmarks. These certain benchmarks will suffice to serve as restrictions outlining High, Medium, and Low risk. Note that we have been given the yearly mean sea level, $\mu$, and the upper $95 \%$ sea level change, $x^{\prime}$, in millimeters per year, so to calculate standard deviation, $\sigma$, we simply equate

$$
\sigma=\left|\frac{\mu-x^{\prime}}{1.96}\right|
$$

Furthermore, given the yearly mean sea level, the cumulative increase in height given as a function of year from 2016, $t$, can be defined as $\int_{0}^{t} \mu \partial t=\mu * t$.

\begin{tabular}{||ccc||}
\hline Site & Mean Sea Level Increase $(\mu)$ & Standard Deviation $(\sigma)$ \\
\hline \hline Acadia National Park & $2.178 \mathrm{~mm} / \mathrm{yr}$ & $0.111 \mathrm{~mm} / \mathrm{yr}$ \\
\hline Cape Hatteras & $3.84 \mathrm{~mm} / \mathrm{yr}$ & $0.628 \mathrm{~mm} / \mathrm{yr}$ \\
\hline Kenai Fjords & $-2.62 \mathrm{~mm} / \mathrm{yr}$ & $0.352 \mathrm{~mm} / \mathrm{yr}$ \\
\hline Olympic National Park & $0.14 \mathrm{~mm} / \mathrm{yr}$ & $0.490 \mathrm{~mm} / \mathrm{yr}$ \\
\hline Padre Island & $3.48 \mathrm{~mm} / \mathrm{yr}$ & $0.383 \mathrm{~mm} / \mathrm{yr}$ \\
\hline
\end{tabular}

According to National Geographic [10], approximately a 1 feet per century increase in sea level poses a dire threat to swamp out coastal regions. As all 5 sites are isles or outlets with minimum sea levels of $0 \mathrm{~m}$ above sea level, similar increases in height would prove devastating and pose a High Risk threat. Therefore, a $>1$ feet per century $(>304.8 \mathrm{~mm})$ increase in sea level would pose a high threat. Continuing on, a $0.3-1$ feet per century (91.44-300.8 $\mathrm{mm}$ ) increase in sea level contributes to hazardous conditions for visitors and wildlife in these national parks that disrupt a park's operations. Thus, these two bounds serve as a benchmark for assigning medium risk to a site. Any increase smaller than either constraint $(>91.44 \mathrm{~mm})$ can be assumed to pose only a low risk.

Now, we now create a three pronged test assigned to distinguish a site's risk rating. Using a normal model, we can calculate the probability of each of the following occuring as a function of time, $t$ for $t>0$. The test uses a normal model with a mean acculumated 
increase in sea level found above to be $\mu * t$ and standard deviation to be $\sigma * \sqrt{t}$ as a repeated sum of $t$ distinct normal models' standard deviation. Similarly, the risk rating assessments will bound the probability of any event occuring by the 3 intervals denoted above. To normalize our data, we multiply through by a factor of $t / 100$ to account for these intervals being bounded by century long limits that validate a risk while our time is only measured in years.

\begin{tabular}{|l|l||}
\hline \multicolumn{2}{|c||}{ Risk Assigning Model } \\
\hline Risk Rating & Test \\
\hline High & $N\left(\mu * t\left[\frac{t}{100}\right], \sigma \sqrt{t}\left[\frac{t}{100}\right]\right) P\left(x>300.8\left[\frac{t}{100}\right]\right)$ \\
Medium & $N\left(\mu * t\left[\frac{t}{100}\right], \sigma \sqrt{t}\left[\frac{t}{100}\right]\right) P\left(300.8\left[\frac{t}{100}\right]>x>91.44\left[\frac{t}{100}\right]\right)$ \\
Low & $\left.N\left(\mu * t\left[\frac{t}{100}\right], \sigma \sqrt{t}\left[\frac{t}{100}\right]\right) P\left(91.44\left[\frac{t}{100}\right]>x\right]\right)$ \\
\hline
\end{tabular}

Substituting a given site's information regarding their mean sea level increase per year and standard deviation, we can find the probability of the site being assigned a High, Medium, or Low Risk rating in $t$ years from now. Of the three tests for a given year, the expression that gives the greatest probability will correspond to the Risk Rating associated with that site. Ergo, if Test 1 gave the greatest probability over the second and third test then the Risk Rating associated with the site can be defined as High.

To illustrate, we take our model into practice using Acadia National Park as an example.

\begin{tabular}{|l|l|l|l|l||}
\hline \multicolumn{5}{|c|}{ Acadia National Park Probability of each Risk Rating } \\
\hline Risk Rating & 10 years & 20 years & 50 years & 100 years \\
\hline High & 0 & 0 & 0 & 0.0001 \\
Medium & $2.0856^{*} 10^{-11}$ & $1.59^{*} 10^{-4}$ & 0.83328 & 0.9998 \\
Low & 1 & 0.9998 & 0.1667 & $1.35^{*} 10^{-8}$ \\
\hline
\end{tabular}

In general, we find that in 10 and 20 years, the risk assessment given to Acadia National Park can be defined as a Low Risk Site. However, progressing into 50 years and 100 years, we shift to deem Acadia a Medium Risk Site.

Applying these tests, we can more generally create a guide predicting the risk of all 5 sites across 10, 20, 50, and 100 years.

\begin{tabular}{|l||l|l|l|l||}
\hline \multicolumn{5}{|c|}{ General Risk Assessments } \\
\hline Site & 10 years & 20 years & 50 years & 100 years \\
\hline Acadia National Park & Low & Low & Medium & Medium \\
\hline Cape Hatteras & Low & Low & Medium & High \\
\hline Kenai Fjords & Low & Low & Low & Low \\
\hline $\begin{array}{l}\text { Olympic National } \\
\text { Park }\end{array}$ & Low & Low & Low & Low \\
\hline Padre Island & Low & Low & Medium & High \\
\hline
\end{tabular}




\section{$2.4 \quad$ Validity of Model and Generalities}

Our model outputs a risk assessment for each location based upon the predicted effect of rising sea levels on the wildlife and the number of visitors the park will have. As a result, we discount the potential effects of the Kenai Fjords decreasing sea level of 2.62 $\mathrm{mm}$ per year. With further analysis, a separate approach could potentially be adapted to find a decreased water supply's effect on ecosystems but ultimately that concern pales in comparison to the catastrophic effects of rising sea levels.

Trivially, we should note that our model only takes meaning with moderate short run periods i.e. from a couple of decades to 2 centuries. As $\lim t \rightarrow 0$, the normal model approaches the Dirac Delta Distribution showcasing no substantive meaning where the normal distribution yields a finding that each site has an equal chance of being High, Medium, and Low Risk. Similarly, as $\lim t \rightarrow \infty$, a similar occurrence of equal probability of each risk occurs according to the model.

In practice, however, when considering small values of $t$ (those of a couple of years), our model finds most sites to be of low risk as expected. As of now, and predicted to be so for the upcoming years, none of the 5 sites are at any immediate concern of flooding [20]. However, for sufficiently large values of $t$, all sites are predicted to be underwater at current estimates and likewise, our model accounts for it. Simple dimensional analysis and finding that regions like Cape Hatteras have been found to be some of the greatest regions at risk of flooding in the U.S. confirm and validate the use of our model [21].

However, applying our model centuries from now begins to display weaknesses of our model. First, we have to consider the limitation of predicting long periods of time from now. Note that the standard deviation is given by,

$$
\sigma\left(\sqrt{t} \frac{t}{100}\right)
$$

Then we have by the 68-95-99.7 rule that roughly $99.7 \%$ of all possible cumulative sea levels in year $t$ is $\pm 3 \sigma\left(\sqrt{t} \frac{t}{100}\right) \mathrm{mm}$ from the mean. If we just consider our greatest value of $\sigma=0.628$, then in 100 years, our model could be off by roughly

$$
3 * .628 * \sqrt{100} \frac{100}{100}=18.84 m m
$$

or roughly 0.74 inches. While this approximation concludes high validity and minimal spread for 100 years into the future, compounding this for possibly tens of centuries can reduce the reliability of our data. Similarly, the ability to discount temperature change as a factor in the change in sea level was only possible because of bounds on short periods of time. Long run approximations of temperature find steady increases in temperature over numerous centuries that would have to be accounted for. Our model is accurate up to about 2 centuries where slowly a significant possibility of error arises. For time periods in between, error is minimized. 


\section{Part 2: Modeling the Effects of Heat Index, Air Quality, and Hurricane Frequency and Intensity on Vulnerability Scores}

\subsection{Restatement of the Problem}

As there are many different climate events that effect NPS coastal park units, we are tasked with the following:

- Develop a mathematical model that assigns a vulnerability score to any coastal National Park and takes into account likelihood and severity of different climate related events.

- Assign a vulnerability score using our model to the five national parks in Part 1.

\subsection{Assumptions}

- Temperature is not included in the model.

Justification: Since the heat index relates temperature and relative humidity, there exists a strong, positive correlation between temperature and heat index. Thus, by analyzing the heat index, the temperature is analyzed in the model as well.

- Wildfires is not included in the model.

Justification: Wildfires can have both a positive and negative effect on a national park. In small amounts, fire is useful because it cleans the debris off the forest floor, makes room for new animals and habitats, kills diseases, and allows select plants to grow [4]. In large amounts on the other hand, wildfires can be extremely costly to extinguish and damage the ecosystems more than they help them. Due to the varying effects of wildfires, we exclude wildfires from our model.

- Kenai Fjords National Park's average temperature and relative humidity are $62^{\circ} \mathrm{F}$ and $97 \%$.

Justification: Since heat index is not measured in Alaska, we used the formula by which heat index is calculated to determine the heat index for Kenai Fjords National Park. Since the temperature was approximately $62^{\circ} \mathrm{F}$, and thus is below $80^{\circ} \mathrm{F}$, we used the formula

$$
\frac{1}{2} \cdot\left(T+61.0+\frac{T-68}{2}+R H \cdot .094\right)
$$

to calculate the heat index. The relative humidity $(\mathrm{RH})$ and temperature $(\mathrm{T})$ were approximated by the city Seward [6] which is 5 miles from the national park.

- The number and severity of hurricanes remains constant for the next 50 years.

Justification Based on previous statistics regarding the number of hurricanes in the past century, there is no statistically significant trend in the number or severity of hurricanes [7]. Hence, for lack of data and simplicity, we will assume the number and severity of hurricanes remains constant for the next 50 years. 


\subsection{Developing the Model}

Our model consists of three factors: heat index $(H I)$, hurricanes $(H)$, and air quality $(A Q I)$. We first established a relationship between the heat index and a Severity Index $\left(S I_{H I}\right)$, as well as between hurricanes and a seperate Severity $\operatorname{Index}\left(S I_{H}\right)$, and between air quality and its own Severity Index $\left(S I_{A Q I}\right)$. Then, after using an adjustment formula to weight the importance of each of the factors, we found the vulnerability $\operatorname{score}(V S)$ by adding the product of the Severity Indices and their respective weights. In order to facilitate the scaling of each of the separate Severity Indices, we set the range for each Severity Index from 0 to 100 .

To find $S I_{H I}$, we used a Four Parameter Logistic Regression, with the minimum attainable value set at 0 and the maximum at 100. Based off of the heat index chart [8], we established references of $80^{\circ} \mathrm{F}, 90^{\circ} \mathrm{F}, 103^{\circ} \mathrm{F}$, and $124^{\circ} \mathrm{F}$ for levels of caution. We gave each of these references a value on the $S I_{H I}$, specifically $(80,25),(90,55),(103,86)$, and $(124,98)$. These estimates were based off of the levels of caution and their likelihood of occurance. These four points thus gave an equation of

$$
S I_{H I}=100-\frac{100}{1+\left(\frac{H I}{88.03121}\right)^{11.46488}}
$$

where $H I$ represents the coastal unit's heat index and $S I_{H I}$ represents the corresponding value on the Severity Index $S I_{H I}$.

We then proceded to model the Severity Index for hurricanes, $S I_{H}$. The danger that hurricanes pose to national parks is based on its intensity and its return period. The intensity, $H$, is directly proportional to the danger that hurricanes pose because the higher intensity of the hurricane, the more damage sustained. The return period is the estimated return period in years for hurricanes passing within 50 nautical miles of various locations on the U.S. Coast [9]. The longer return period for a hurricane, the lesser threat that hurricanes pose to that region. Thus, we obtain the equation

$$
S I_{H}=\frac{k \cdot H}{\tau},
$$

where $\tau$ is the return period and $k$ is a constant.

There are 5 classifications of hurricanes: H1, H2, H3, H4, and H5, with $\mathrm{H} 5$ being the most severe. There are two classifications of tropical storms: TS (Tropical storm) and TD (Tropical depression). There exists a third type of storm known as a ET, or extratropical storm. ETs can be any intensity, ranging from H5 to TD. We first assign each storm other than ET a value from 1-7, as shown in the following table.

\begin{tabular}{||ccccccc||}
\hline TD & TS & H1 & H2 & H3 & H4 & H5 \\
\hline 1 & 2 & 3 & 4 & 5 & 6 & 7 \\
\hline
\end{tabular}

To assign a value to ET, we found the likelihood of each type of hurricane or tropical storm occuring, then multiplied the likelihoods by the assigned value to find the expected value of the storms. There were 856.8 tropical storms or depressions in the past century 
[11], and assuming that there were an equal number of tropical storms and depressions due to lack of data, we get a value of $856.8 \cdot 15=1285.2$ for tropical storms. Adding the hurricanes multiplied by their respective values, we get an expected value of

$$
E T=\frac{856.8 \cdot 1.5+199 \cdot 3+113 \cdot 4+97 \cdot 5+20 \cdot 6+5 \cdot 7}{856.8+199+113+97+20+5}=2.3059 .
$$

Now that we have assigned a value to ET, we calculate $H$ to be the expected value of the hurricanes:

$$
H=\frac{2.3059 \cdot E T+1 \cdot T D+2 \cdot T S+3 \cdot H 1+4 \cdot H 2+5 \cdot H 3+6 \cdot H 4+7 \cdot H 5}{E T+T D+T S+H 1+H 2+H 3+H 4+H 5}
$$

where ET, TD, TS, H1, H2, H3, H4, and H5 represent the number of hurricanes of those classifications that hit the coastal unit within a given year. Since

$$
H \leq \tau
$$

which is because the return period is greater than 5 and $H$ is less than 5 , we set $k=100$ to scale $S I_{H}$ from 0 to 100 . Note that although it is possible for $H$ to be greater than 5 in theory, it is not possible in reality. The number of ET, TS, TD, H1, and H2 storms significantly outnumber the number of $\mathrm{H} 3, \mathrm{H} 4$, and $\mathrm{H} 5$ storms, which brings $H \leq 5$. This gives us the final model for hurricanes:

$$
S I_{H}=\frac{100 \cdot H}{\tau} .
$$

The last Severity Index is for the air quality, which is measured using the AQI, or air quality index. We use the variables Average AQI Value $(\mu)$, Average Maximum AQI $(\bar{M})$, and Average Minimum AQI $(\bar{m})$. Our base model for $S I_{A Q I}$ is

$$
S I_{A Q I}=k \cdot \mu+j \cdot \bar{M}+i \cdot \bar{m}
$$

where $k, j$, and $i$ are constants. In order to average the AQI, we take $k=2, j=1$, and $i=1$. Based on the data provided, $0 \leq \mu \leq 70,0 \leq \bar{M} \leq 100$, and $0 \leq \bar{m} \leq 30$. The maximum value of $S I_{A Q I}$ is thus $2 \cdot 70+100+30=270$, so in order to scale it down to 100 , we divide $k, j$, and $i$ by 2.7 to get $k=7.4, j=.37$, and $i=.37$. This results in

$$
S Q_{A Q I}=.74 \cdot \mu+.37 \cdot \bar{M}+.37 \cdot \bar{m} .
$$

\subsection{Putting it all together}

Now that we have $S I_{H I}, S I_{H}$, and $S I_{A Q I}$, we can finally weight these to obtain an equation for $V S$, the vulnerability score. We assign weights based on the severity of the threat as well as the likelihood of the threat. Since the heat index, and thus temperature, is rated at the highest relative threat, with hurricanes and thus flooding following, and air quality trailing in last [12], we assign values of $\frac{1}{2}, \frac{3}{10}$, and $\frac{1}{5}$ to $S I_{H I}, S I_{H}$, and $S I_{A Q I}$. 
However, since hurricanes are extremely rare in the West Coast, we adjust the weights of hurricanes and air pollution for coastal units in the West to $\frac{1}{20}$ and $\frac{9}{20}$ respectively. Thus, our vulnerability score model is:

$$
V S=\left[\begin{array}{lll}
.5 & .3 & .2
\end{array}\right] \cdot\left[\begin{array}{c}
S I_{H I} \\
S I_{H} \\
S I_{A Q I}
\end{array}\right]
$$

for coastal units in the East Coast, and

$$
V S=\left[\begin{array}{lll}
.5 & .05 & .45
\end{array}\right] \cdot\left[\begin{array}{c}
S I_{H I} \\
S I_{H} \\
S I_{A Q I}
\end{array}\right]
$$

for coastal units in the West Coast.

\subsection{Verifying the Model}

We used this model to assign vulnerability scores to the five national parks, and then assessed the conditions of those five parks with the vulnerability scores to determine its accuracy.

\begin{tabular}{||ccccccc||}
\hline Site & $H I$ & $H$ & $\tau$ & $\mu$ & $\bar{M}$ & $\bar{m}$ \\
\hline \hline Acadia National Park & 85.61 & 2.306 & 34 & 41.53 & 84.77 & 23.39 \\
\hline Cape Hatteras & 87.66 & 2.395 & 7 & 43.22 & 82.34 & 20.36 \\
\hline Kenai Fjords & 62.78 & 0 & N/A & 31.8 & 66.12 & 12.59 \\
\hline Olympic National Park & 81.52 & 0 & N/A & 35.46 & 58.56 & 19.35 \\
\hline Padre Island & 92.01 & 2.545 & 15 & 44.63 & 95.05 & 21.92 \\
\hline
\end{tabular}

We then computed the severity indices, getting the following scores.

\begin{tabular}{||ccccc||}
\hline Site & $S I_{H I}$ & $S I_{H}$ & $S I_{A Q I}$ & $V S$ \\
\hline \hline Acadia National Park & 42.06 & 6.782 & 70.828 & 50.04 \\
\hline Cape Hatteras & 48.78 & 34.21 & 70.06 & 48.70 \\
\hline Kenai Fjords & 2 & 0 & 52.72 & 23.724 \\
\hline Olympic National Park & 29.31 & 0 & 55.12 & 39.46 \\
\hline Padre Island & 62.40 & 16.97 & 76.38 & 51.57 \\
\hline
\end{tabular}

Since the two regions were weighted differently, the bounds for the point in which the vulnerability score becomes critical are different. For the East Coast, $V S_{\text {bound }}=50$; while for the West Coast, $V S_{\text {bound }}=40$. In order to make comparisons, we used an adjusted vulnerability score, $V S^{*}$. We calculated this by dividing the unit's $V S$ by the bound for the vulnerability score in that region. The table below shows the adjusted vulnerability scores.

\begin{tabular}{||cc||}
\hline Site & $V S^{*}$ \\
\hline \hline Acadia National Park & 1.0008 \\
\hline Cape Hatteras & 0.974 \\
\hline Kenai Fjords & 0.5931 \\
\hline Olympic National Park & 0.9865 \\
\hline Padre Island & 1.0314 \\
\hline
\end{tabular}


If $V S^{*}$ for a coastal unit is greater than one, then it has surpassed the bound, and thus is in a critical state. If $V S^{*} \leq 1$, then the closer it is to 1 , the worse condition that the park is in. Thus, according to our model, Padre Island is in the worst condition, followed by Acadia National Park, both of which are critical. Cape Hatteras and Olympic National Park are close to critical, and Kenai Fjords is still in good condition.

Padre Island flooded in 2015 as well as 2014, which verifies the fact that it is in critical condition. Acadia is a Class 1 Area under the Clean Air Act, which shows that it is in need of the highest air quality protection [18]. The unusually high air pollution in Acadia confirms that it is also in a critical state, further verifying our model. While Olympic National Park and Cape Hatteras do not have critical issues in the status quo, both parks have projected climate events to be a dire issue in the near future. Because both parks fear climate-related events to be a prominent issue in the near future, this confirms that both parks are still safe but close to critical. Kenai Fjords is still in good condition, thus completing the validation of our model.

In order to apply this model for other NPS coastal units, the following information is necessary:

- Heat Index

If the heat index is not available, as was the case for Kenai Fjords National Park, then the average temperature and relative humidity are necessary.

\section{- Hurricanes}

The intensity of each of the hurricanes and the frequency of the hurricanes is necessary.

\section{- Air Quality}

The average, average maximum, and average minimum AQI in the current year is necessary

With this information, the Severity Index for that site can be calculated, and depending on whether or not the coastal unit is in the East or West Coast, the weights would be different. Then, the $V S$ would be calculated for the NPS coastal unit, and then using the respective benchmark would give the current state of the national park.

\subsection{Future Projections}

After assigning vulnerability scores to each of the different parks, we were curious as to when the two parks, Cape Hatteras and Olympic National Park, would enter a critical state. To do so, we projected the heat index and AQI, and kept hurricanes constant according to Assumption 4.

Using the data provided, we created a logarithmic curve of best fit for the AQI and a linear line of best fit for the heat index. We used a linear model for projecting heat index because the linear model with $95 \%$ confidence intervals showed a statistically significant trend at the $95 \%$ confidence level for heat waves in India [22]. Although we were 
concerned about the heat indices in the United States, the trends for heat indices are of the same type throughout the world. For example, if a logarithmic curve was the general trend, then the heat indices would follow a logarithmic curve in the United States. The logarithmic model was chosen for projecting AQI because the variance was the highest out of all of the different models.

For the heat index, we took the provided data and applied a linear model. We calculated the following equations for four out of the five national parks (Kenai Fjords was excluded due to lack of data).

\begin{tabular}{||cc||}
\hline Site & Line of Best Fit \\
\hline \hline Acadia National Park & $H I=.0074 t+80.841$ \\
\hline Cape Hatteras & $H I=-.004 t+83.821$ \\
\hline Olympic National Park & $H I=.0023 t+79.529$ \\
\hline Padre Island & $H I=.0036 t+91.552$ \\
\hline
\end{tabular}

These equations are for the four specific national parks, so to estimate the heat index for other national parks, we averaged these equations to obtain

$$
H I=.00279 t+83.94
$$

where $t$ represents the number of years that we are projecting. Converting to the Severity Index, we get

$$
S I_{H I}=100-\frac{100}{1+\left(\frac{.00279 t+83.94}{88.03121}\right)^{11.46488}} \text {. }
$$

For the AQI, since we used the average $(\mu)$, average maximum $(\bar{M})$, and average minimum $(\bar{m})$, we projected all of these values, then combined them to obtain a model projecting AQI.

\begin{tabular}{||cccc||}
\hline Site & $\mu$ & $\bar{M}$ & $\bar{m}$ \\
\hline \hline Acadia NP & $\mu=-6.3 \ln t+107.9$ & $\bar{M}=-1.7 \ln t+45$ & $\bar{m}=4.8 \ln t+22.4$ \\
\hline Cape Hatteras & $\mu=-4.7 \ln t+68.2$ & $\bar{M}=-2.9 \ln t+46.5$ & $\bar{m}=-1.6 \ln t+22.9$ \\
\hline Kenai Fjords & $\mu=-5.1 \ln t+76.5$ & $\bar{M}=-3.2 \ln t+32.6$ & $\bar{m}=-1.6 \ln t+15.9$ \\
\hline Olympic NP & $\mu=-9.9 \ln t+92$ & $\bar{M}=1.4 \ln t+32.6$ & $\bar{m}=3.6 \ln t+.2$ \\
\hline Padre Island & $\mu=-7.4 \ln t+100$ & $\bar{M}=1.2 \ln t+42.1$ & $\bar{m}=2.1 \ln t+17.6$ \\
\hline
\end{tabular}

Again, since these equations are specific for the different sites, we averaged these equations to obtain a general equation for $\mu, \bar{M}$, and $\bar{m}$ :

$$
\begin{gathered}
\mu=-6.68 \ln t+88.92, \\
\bar{M}=-1.01 \ln t+40.89, \\
\bar{m}=.58 \ln t+18.19 .
\end{gathered}
$$

Thus, our general model for $S I_{A Q I}$ is

$$
S I_{A Q I}=-3.44 \ln t+69.96 .
$$

Combined with the constant $S I_{H}$, we can thus project $V S$. 


\section{Part 3: Profitability and Vulnerability}

\subsection{Restatement of the Problem}

As the NPS only has a limited amount of resources and budget to split among its many sites, we are tasked with deciding just how much each site will receive in financial backing. Our task consists of two parts:

- Develop a mathematical model that predicts the number of visitors in each park as a function of time, taking into account the adjusted vulnerability score.

- Using the output of our model that predicts the future number of visitors to decide how much each site will receive in financial resources.

\subsection{Analysis of the Problem}

When considering the factors that affect the number of visitors to a given site, there are a number of unknown variables like the economic state of the nation at that time and unpredictable natural disasters like earthquakes and tornadoes that we cannot account for. Factors that we can model more accurately are the general popularity of the specific site, which is reflected by trend of the number of visitors, along with the environmental state of the site, which is reflected through the adjusted vulnerability score.

\subsection{Assumptions}

- There is a fixed budget for the money that is assigned to be distributed among the 5 sites.

Justification: Although the problem only concerns 5 sites, in reality the NPS deals with far more sites. However, we must assume the NPS has a fixed budget so that the model can generate concrete values.

- Unless adjusted vulnerability is higher than a certain threshold, it plays no role in affecting the the number of visitors per year.

Justification: Although a high adjusted vulnerability can affect a visitor's decision to attend a park, the visitor will not care about the difference between two relatively low adjusted vulnerability scores.

- The benchmark adjusted vulnerability score is 1.

Justification: A benchmark adjusted vulnerability score of 1 is not justified but is necessary in order to analyze the impact of vulnerability on $v(t)$.

- Growth in number of visitors each year for a given site is approximately linear.

Justification: The population of the United States is growing at an exponential rate; however, with the increasing immersion on technology and the virtual world, there is decreasing interest in nature and national parks among the general populace. 
- The two main factors in determining the amount of money that should be allocated to a site are adjusted vulnerability score and number of visitors.

Justification: Adjusted vulnerability score represents the environmental state of the park which helps us determine how likely it is that the park will need funding for climate-related events. The number of visitors represents the magnitude of potential change the funding a park can accomplish, because we want the funds to reach to as many people as possible and generate revenue for the park.

- The number of visitors is more important to consider than adjusted vulnerability score.

Justification: While the goal of the NPS is to enhance the natural experience as much as possible, earning revenue is much more essential in securing a substantial budget for future years. Therefore, it is imperative that the number of visitors is emphasized more so that more entrance revenue is generated.

- We use the predicted adjusted vulnerability scores of the sites in 20 years rather than the present adjusted vulnerability scores to determine how the NPS should allocate funds.

Justification: To account for long-term changes in the adjusted vulnerability of the site, and better fund the sites that will be changing significantly over time, we take into account the future adjusted vulnerability of a site rather than the current adjusted vulnerability. Our model is based on a linear regression with a low $R^{2}$ value, so it will not hold for long periods of time. The model will hold for about 20 years, but not much longer.

\subsection{Developing the Model}

The first part of our process consists of developing a model representing the change in number of visitors for a given site. We use a linear regression for the model as it fits the data better than regressions of higher degrees. However, we must also take into account the impact of high adjusted vulnerability on a visitor's decision to travel to a park. Thus, our equation is as follows:

$$
v(t)= \begin{cases}a t+b-k\left(V S_{\text {site }}^{*}-V S_{\text {bench }}^{*}\right) & V S_{\text {bench }}^{*} \leq V S_{\text {site }}^{*} \\ a t+b & V S_{\text {bench }}^{*} \geq V S_{\text {site }}^{*}\end{cases}
$$

where $a$ and $b$ are constants to be determined through the data we collected [13], different for each site, $V S_{\text {bench }}^{*}=1, k=1,000,000$ and $V S_{\text {site }}^{*}$ is the adjusted vulnerability of the site. When $V S_{\text {bench }}^{*} \geq V S_{\text {site }}^{*}$, the adjusted vulnerability is no longer a factor in determining the number of visitors. 


\section{Kenai Fjords Annual Visitors per Year $(t=0$ corresponds to 2004)}

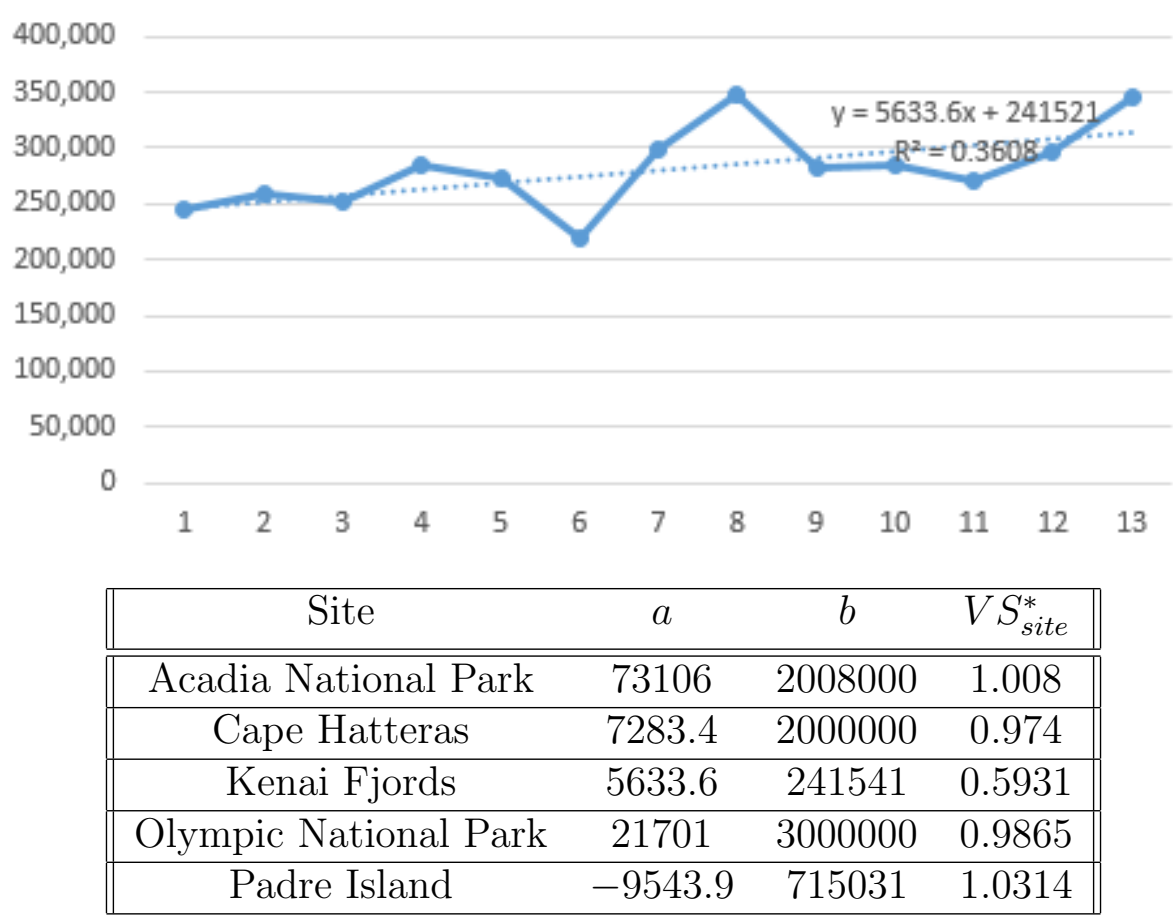

Using $v(t)$, we calculate the visitor index by dividing $v(t)$ by the $v(t)$ of the site with the greatest $v(t)$. We use $t=20$, as again, the model will have inaccuracies in the long run.

$$
v(20)_{\text {index }}=\frac{v(20)_{\text {site }}}{\max \left(v(20)_{i}\right)}
$$

We normalize the visitor indices by using the predicted maximum number of visitors across all five sites in 20 years. This allows us to compare each site relative to one another based on the predicted number of visitors in 20 years. However, the predicted maximum number of visitors changes each year, and as a result, using a different year would lead to different visitor indices for each site. This would also change how funds should be distributed between the five parks. As long as the visitor indices do not change dramatically in other years, the indices calculated using the predicted number of visitors in 20 years will be reasonable to use in determining how the NPS should distribute funding. However, if the visitor indices differ drastically in other years, it would be necessary to consider visitor indices for multiple years when calculating how funds should be distributed.

From the adjusted vulnerability score we determined for each of the sites in Part 2 of this problem, we calculate an adjusted vulnerability index which is defined by the adjusted vulnerability score of current site divided by the adjusted vulnerability score of the site with the greatest adjusted vulnerability:

$$
V S_{\text {index }}^{*}=\frac{V S_{\text {site }}^{*}}{\max \left(V S_{i}^{*}\right)}
$$

Once we determine the adjusted vulnerability index and visitor index of each site, we can define that site's financial utility by multiplying .75 by the visitor index and .25 by 
the adjusted vulnerability index and adding those two numbers up. Note that the sum should still be a number in between 0 and 1 . Once we calculate the financial utility for each site, we can calculate the percentage of funds allocated to each site as follows:

$$
\text { Percentage of Funds }=\frac{F_{\text {site }}}{\sum\left(F_{i}\right)}
$$

where $F$ stands for financial utility.

\begin{tabular}{||ccc||}
\hline Site & Financial Utility & Percentage of Funds \\
\hline \hline Acadia National Park & 0.9943 & $30.48 \%$ \\
\hline Cape Hatteras & 0.7009 & $21.49 \%$ \\
\hline Kenai Fjords & 0.2876 & $8.82 \%$ \\
\hline Olympic National Park & 0.9219 & $28.27 \%$ \\
\hline Padre Island & 0.3567 & $10.94 \%$ \\
\hline
\end{tabular}

\subsection{Justification and Discussion}

The model heavily relies on weighted averages since in reality, the finances should take precedence over preservation because finances determine potential for future actions. With an enlarged budget, the NPA is able to further fund projects that would've otherwise not been possible.

Moreover, the $v(t)$ function was designed to model the visitor growth; however, based on the data that we had, the linear regression equation had a low $R^{2}$ value. There was no apparent pattern to the number of visitors per year in each site because they fluctuated randomly. Thus, although our model has some inaccuracies, we believe that it represented the data more fairly than most other models could have.

In addition, we ensured that all the national parks received a non-negligible amount of funding in this case, and even in other non-trivial cases each site should get significant funding.

Testing this model for accuracy is difficult, because it would require coming up with a way to measure the success. In addition, even in the case that there was some measure of success, we would have to collect data over the future years.

The first part of our model, the equation of visitors against time is quite sensitive to assumptions. We had to make many assumptions in order to implement the model in the first place because of the sporadic nature of the visitor data. In addition, the model does hold for long periods of time because of this. 


\section{Conclusion}

\section{$5.1 \quad$ Strengths}

- Our model addresses a variety of criteria that have significant influences on sea level, safety, visitor count, and the distribution of funding.

- Our model outputs a number of intermediate values, making troubleshooting easier when presented with faulty errors.

- Our model relied heavily on prior data and utilizing that data to make projections for the future which lends credibility.

- Assuming we have sufficient information, our models can be extended to more national parks.

\subsection{Weaknesses}

- While multiple intermediate steps makes troubleshooting easier, there would be more steps to rework in the case of an early error.

- Our projections were not completely correlated with the data (moderately low $R^{2}$ values).

- Our third model made a significant number of assumptions which while not logically incorrect may introduce unaccounted variation.

- The first and second models we developed cannot be reasonably extended to more than one century. 


\section{References}

[1] "Sea Level Trends - NOAA Tides and Currents." Sea Level Trends - NOAA Tides and Currents. N.p., n.d. Web. 26 Feb. 2017.

https://tidesandcurrents.noaa.gov/sltrends/sltrends.html

[2] "Climate Change Indicators: Sea Level." EPA. Environmental Protection Agency, 17 Dec. 2016. Web. 26 Feb. 2017.

https://www.epa.gov/climate-indicators/climate-change-indicators-sea-level

[3] "14. How Fast Is Sea Level Rising?" Royal Society. N.p., n.d. Web. 26 Feb. 2017.

https://royalsociety.org/topics-policy/projects/climate-change-evidence-causes/question$14 /$

[4] California, State Of. "Hot Topics." Cal Fire. N.p., n.d. Web. 26 Feb. 2017. http://calfire.ca.gov/communications/downloads/fact_sheets/TheBenefitsofFire.pdf

[5] "Heat Index Equation." Weather Prediction Center. N.p., n.d. Web. 26 Feb. 2017. http://www.wpc.ncep.noaa.gov/html/heatindex_equation.shtml

[6] "WeatherSpark Beta." Average Weather For Seward, Alaska, USA - WeatherSpark. N.p., n.d. Web. 26 Feb. 2017.

https://weatherspark.com/averages/33089/Seward-Alaska-United-States

[7] "TCFAQ E11) How Many Tropical Cyclones Have There Been Each Year in the." Atlantic Oceanographic and Meteorological Laboratories. N.p., n.d. Web. 26 Feb. 2017. http://www.aoml.noaa.gov/hrd/tcfaq/E11.html

[8] US Department of Commerce, NOAA, National Weather Service. "National Weather Service." National Weather Service. NOAA's National Weather Service, n.d. Web. 26 Feb. 2017.

https://www.weather.gov

[9] "Top 5 Most Vulnerable U.S. Cities to Hurricanes." Climate Central. N.p., 06 June 2012. Web. 26 Feb. 2017.

http://www.climatecentral.org/news/top-5-most-vulnerable-us-cities-to-hurricanes [10] Warne, Photograph By Kennedy. "Sea Level Rise." National Geographic. N.p., 24 Feb. 2017. Web. 26 Feb. 2017.

http://www.nationalgeographic.com/environment/global-warming/sea-level-rise/

[11] "Landfalling Hurricane Probability Project." Landfalling Hurricane Probability Project. N.p., n.d. Web. 26 Feb. 2017.

http://www.e-transit.org/hurricane/welcome.html [12] Photograph by Michael Melford, National Geographic. "Top Ten Issues Facing the National Parks - National Geographic." Top Ten Issues Facing the National Parks - National Geographic. N.p., 24 Feb. 2017. Web. 26 Feb. 2017.

http://www.nationalgeographic.com/travel/top-10/national-parks-issues/

[13] "NPS Stats." National Parks Service. U.S. Department of the Interior, n.d. Web. 26 Feb. 2017.

https://irma.nps.gov/Stats/

[14] "Saffir-Simpson Hurricane Wind Scale." Saffir-Simpson Hurricane Wind Scale. N.p., n.d. Web. 26 Feb. 2017.

http://www.nhc.noaa.gov/aboutsshws.php

[15] "Hurricanes." Hurricanes. N.p., n.d. Web. 26 Feb. 2017.

https://coast.noaa.gov/hurricanes/ 
[16] "About Air Data Reports." EPA. Environmental Protection Agency, 14 Sept. 2016. Web. 26 Feb. 2017.

https://www.epa.gov/outdoor-air-quality-data/about-air-data-reports\#aqidaily

[17] "Air Quality Index Daily Values Report." EPA. Environmental Protection Agency, 04 Jan. 2017. Web. 26 Feb. 2017.

https://www.epa.gov/outdoor-air-quality-data/air-quality-index-daily-values-report [18] "Air Quality." National Parks Service. U.S. Department of the Interior, n.d. Web. 26 Feb. 2017.

https://www.nps.gov/acad/learn/nature/airquality.htm

[19] US Department of Commerce, National Oceanic and Atmospheric Administration. "Is Sea Level Rising?" NOAA's National Ocean Service. N.p., 27 Oct. 2008. Web. 26 Feb. 2017.

http://oceanservice.noaa.gov/facts/sealevel.html

[20] "Which Coastal Cities Are at Highest Risk of Damaging Floods? New Study Crunches the Numbers." World Bank. N.p., n.d. Web. 26 Feb. 2017.

http://www.worldbank.org/en/news/feature/2013/08/19/coastal-cities-at-highest-risk-floods [21] Carolina, CBS North. "Record Flooding at NC Outer Banks as Hermine's Exit Causes Unexpected Problems." WNCN. N.p., 03 Sept. 2016. Web. 26 Feb. 2017.

http://wncn.com/2016/09/03/record-flooding-at-nc-outer-banks-as-hermines-exit-causesunexpected-problems/

[22] Nature News. Nature Publishing Group, n.d. Web. 26 Feb. 2017.

http://www.nature.com/articles/srep26153/figures/2 